\title{
Global Stability for a Viral Infection Model with Saturated Incidence Rate
}

\author{
Huaqin Peng ${ }^{1}$ and Zhiming Guo ${ }^{1,2}$ \\ ${ }^{1}$ School of Mathematics and Information Sciences, Guangzhou University, Guangzhou 510006, China \\ ${ }^{2}$ Key Laboratory of Mathematics and Interdisciplinary Science of Guangdong, Higher Education Institutes, Guangzhou University, \\ Guangzhou 510006, China
}

Correspondence should be addressed to Zhiming Guo; gzm100@sina.com

Received 13 January 2014; Accepted 18 February 2014; Published 6 April 2014

Academic Editor: Chuangxia Huang

Copyright (C) 2014 H. Peng and Z. Guo. This is an open access article distributed under the Creative Commons Attribution License, which permits unrestricted use, distribution, and reproduction in any medium, provided the original work is properly cited.

A viral infection model with saturated incidence rate and viral infection with delay is derived and analyzed; the incidence rate is assumed to be a specific nonlinear form $\beta x v /(1+\alpha v)$. The existence and uniqueness of equilibrium are proved. The basic reproductive number $R_{0}$ is given. The model is divided into two cases: with or without delay. In each case, by constructing Lyapunov functionals, necessary and sufficient conditions are given to ensure the global stability of the models.

\section{Introduction}

In recent years, study of infectious disease model has been a hot issue; the main cause of infectious disease is the virus invasion. As we know, viral cytopathicity within target cells is very common. A number of mathematical models have been used to study virus dynamics. In 1996, Nowak et al. [1] designed a simple but natural mathematical model based on ordinary differential equation. The model is as follows:

$$
\begin{aligned}
& \frac{d x(t)}{d t}=\lambda-d x(t)-\beta x(t) v(t), \\
& \frac{d y(t)}{d t}=\beta x(t) v(t)-a y(t), \\
& \frac{d v(t)}{d t}=\kappa y(t)-\gamma v(t),
\end{aligned}
$$

where $x(t)$ denotes the number of uninfected cells, $y(t)$ the numbers of infected cells, and $v(t)$ the numbers of free viral particles at time $t$, respectively. In model (1), uninfected target cells are assumed to be produced at a constant rate $\lambda$ and died at rate $d x$. Infection of target cells by in-host free viruses is assumed to occur at a bilinear rate $\beta x v$; infected cells are lost at a rate $a y$. Free viruses are produced by infected cells at a rate $\kappa y$, in which $\kappa$ is the average number of viral particles produced over the lifetime of a single infected cell. Free viral particles die at a rate $\gamma v$. For model (1), Korobeinikov [2] established the condition of global stability in 2004 . Some other viral dynamical models were proposed by later researchers; see for example [3-8].

In [7], Wodarz and Levy pointed out that the term $a y(t)$ in model (1) should consist of two parts: one is the natural death of infected cells, the other is viral cytopathicity. In 2012, Li et al. [4] assumed that infected cells burst and then release viral particles (i.e., viral cytopathicity occurs) after uninfected cells were infected by a constant period of time $\tau$; that is, the time period of viral cytopathicity within target cells is $\tau$. They incorporated the delay of viral cytopathicity within target cells and built a new model:

$$
\begin{aligned}
& \frac{d x(t)}{d t}=\lambda-d x(t)-\beta x(t) v(t), \\
& \frac{d y(t)}{d t}=\beta x(t) v(t)-\beta e^{-d \tau} x(t-\tau) v(t-\tau)-d y(t), \\
& \frac{d v(t)}{d t}=\kappa \beta e^{-d \tau} x(t-\tau) v(t-\tau)-\gamma v(t) .
\end{aligned}
$$

By constructing Lyapunov functionals, necessary and sufficient conditions were obtained ensuring the global stability of the model. 
In models (1) and (2), the researcher studied the viral dynamics with bilinear incidence rate $\beta x v$. As we know, as the viral particles diffuse in the body, the person often takes some actions when $v$ gets large. In order to describe the inhibitory effect from the uninfected cells when the number of viral cytopathicity is large enough, following the idea of [9], we propose an incidence rate $\beta x v /(1+\alpha v)$, where $\beta v$ measures the infection force of the viral, and $\alpha$ reflects the level of inhibitory action.

Similar to the discussions in [4], we assume that the viral cytopathicity has time delay. When the delay of viral cytopathicity within target cells is $\tau$ and the natural death rate of per target cell is $d$, the number of infected cells at time $t(t>\tau)$ can be represented by

$$
y(t)=\int_{t-\tau}^{t} \frac{\beta x(\theta) v(\theta)}{1+\alpha v(\theta)} e^{-d(t-\theta)} d \theta, \quad \text { for } t>\tau,
$$

where $e^{-d(t-\theta)}$ is the probability that target cells survive from time $\theta$ to time $t$, and $(\beta x(\theta) v(\theta) /(1+\alpha v(\theta))) e^{-d(t-\theta)}$ is the number of target cells being infected at time $\theta$ and still surviving at time $t$.

Differentiating $y(t)$ of (3), we get

$$
\begin{aligned}
\frac{d y(t)}{d t}= & -d \int_{t-\tau}^{t} \frac{\beta x(\theta) v(\theta)}{1+\alpha v(\theta)} e^{-d(t-\theta)} d \theta+\frac{\beta x(t) v(t)}{1+\alpha v(t)} \\
& -e^{-d \tau} \frac{\beta x(t-\tau) v(t-\tau)}{1+\alpha v(t-\tau)} \\
= & \frac{\beta x(t) v(t)}{1+\alpha v(t)}-\beta e^{-d \tau} \frac{x(t-\tau) v(t-\tau)}{1+\alpha v(t-\tau)}-d y(t),
\end{aligned}
$$

where $\beta e^{-d \tau}(x(t-\tau) v(t-\tau) /(1+\alpha v(t-\tau)))$ is the transfer rate of the infected cells being used to produce free viruses at time $t$; the recruitment rate of free virus at time $t$ is $\kappa \beta e^{-d \tau}(x(t-$ $\tau) v(t-\tau) /(1+\alpha v(t-\tau)))$, in which $\kappa$ is the average number of viral particles produced by an infected target cell when viral cytopathicity occurs, which implies that the recruitment of virus at time $t$ depends on the number of target cells that were newly infected at time $t-\tau$ and still alive at time $t$. Therefore following the model (2), we obtain a basic viral dynamical model of delay differential equations:

$$
\begin{aligned}
& \frac{d x(t)}{d t}=\lambda-d x(t)-\frac{\beta x(t) v(t)}{1+\alpha v(t)}, \\
& \frac{d y(t)}{d t}=\frac{\beta x(t) v(t)}{1+\alpha v(t)}-\beta e^{-d \tau} \frac{x(t-\tau) v(t-\tau)}{1+\alpha v(t-\tau)}-d y(t), \\
& \frac{d v(t)}{d t}=\kappa \beta e^{-d \tau} \frac{x(t-\tau) v(t-\tau)}{1+\alpha v(t-\tau)}-\gamma v(t) .
\end{aligned}
$$

Since the variable $y$ does not appear in the first and the third equations of (5), we only focus on the following equations:

$$
\begin{aligned}
& \frac{d x(t)}{d t}=\lambda-d x(t)-\frac{\beta x(t) v(t)}{1+\alpha v(t)}, \\
& \frac{d v(t)}{d t}=\kappa \beta e^{-d \tau} \frac{x(t-\tau) v(t-\tau)}{1+\alpha v(t-\tau)}-\gamma v(t),
\end{aligned}
$$

which has the same dynamics with system (5).
Let $b=\kappa e^{-d \tau}$, by (6), we have

$$
\begin{aligned}
& \frac{d x(t)}{d t}=\lambda-d x(t)-\frac{\beta x(t) v(t)}{1+\alpha v(t)} \\
& \frac{d v(t)}{d t}=\beta b \frac{x(t-\tau) v(t-\tau)}{1+\alpha v(t-\tau)}-\gamma v(t),
\end{aligned}
$$

where all the parameters are assumed to be positive.

The rest of this paper is organized as follows. In the next section we will derive the infection-free equilibrium and the infection equilibrium. In Section 3, we carry out a qualitative analysis of the model, and stability conditions for the infection-free equilibrium and the infection equilibrium are derived, respectively. A brief conclusion will be given in Section 4.

\section{Positive Solutions and Equilibria}

Due to the biological meaning of the components $(x(t), v(t))$, we consider system (7) with the following initial conditions:

$$
\begin{aligned}
\frac{d x(t)}{d t} & =\lambda-d x(t)-\frac{\beta x(t) v(t)}{1+\alpha v(t)}, \\
\frac{d v(t)}{d t} & =\beta b \frac{x(t-\tau) v(t-\tau)}{1+\alpha v(t-\tau)}-\gamma v(t), \\
x(\theta) & =\varphi_{1}(\theta) \geq 0, \varphi_{1}(0)>0, \quad \theta \in[-\tau, 0], \\
v(\theta) & =\varphi_{2}(\theta) \geq 0, \varphi_{2}(0)>0, \quad \theta \in[-\tau, 0] .
\end{aligned}
$$

Equation (8) is a system of retarded differential equations in $C=C\left([-\tau, 0], \mathbb{R}^{2}\right) . C$ is a Banach space of continuous mappings from $[-\tau, 0]$ into $\mathbb{R}^{2}$ with norm $\|\psi\|=\sup _{-\tau \leq \theta \leq 0}|\psi(\theta)|$ for $\psi \in C$. We denote

$$
\begin{array}{r}
C^{+}=\left\{\left(\varphi_{1}, \varphi_{2}\right) \in C \mid \varphi_{1}(0)>0, \varphi_{2}(0)>0,\right. \\
\left.\varphi_{1}(\theta) \geq 0, \varphi_{2}(\theta) \geq 0, \theta \in[-\tau, 0]\right\} .
\end{array}
$$

As usual, for any continuous function $x \in C([-\tau,+\infty), \mathbb{R})$ and any given $t \geq 0, x_{t}$ is defined as $x_{t} \in C([-\tau, 0] \mathbb{R}), x_{t}(\theta)=$ $x(t+\theta)$, for any $\theta \in[-\tau, 0]$.

Theorem 1. All the solutions $(x(t), v(t))^{T}$ of (8) under the initial conditions are positive on $[0, \infty)$.

Proof. Assume that there is a $t_{1}\left(t_{1}>0\right)$ such that $x\left(t_{1}\right)=0$; then by $x(0)>0$ and the continuity of $x$, there is $t^{*}=\inf \{t>$ $0, x(t)=0\}>0$ such that $x(t)>0$ for $t \in\left[0, t^{*}\right)$. Then we have $x^{\prime}\left(t^{*}\right) \leq 0$. However, $x^{\prime}\left(t^{*}\right)=\lambda>0$ by the first equation of (8); this is a contradiction. Therefore $x(t)>0$ for all $t>0$.

From the second equation of (8)

$$
\frac{d v(t)}{d t}=\beta b \frac{x(t-\tau) v(t-\tau)}{1+\alpha v(t-\tau)}-\gamma v(t)
$$


Multiplying $e^{\gamma t}$ in both sides of the above equation and integrating it from 0 to $t$, we have

$$
\begin{gathered}
\int_{0}^{t} e^{\gamma \theta} d v(\theta)+\int_{0}^{t} e^{\gamma \theta} \gamma v(\theta) d \theta \\
=\int_{0}^{t} e^{\gamma \theta} \beta b \frac{x(\theta-\tau) v(\theta-\tau)}{1+\alpha v(\theta-\tau)} d \theta, \\
e^{\gamma t} v(t)-v(0)=\int_{0}^{t} e^{\gamma \theta} \beta b \frac{x(\theta-\tau) v(\theta-\tau)}{1+\alpha v(\theta-\tau)} d \theta, \\
v(t)=\left[v(0)+\int_{0}^{t} e^{\gamma \theta} \beta b \frac{x(\theta-\tau) v(\theta-\tau)}{1+\alpha v(\theta-\tau)} d \theta\right] e^{-\gamma t} .
\end{gathered}
$$

Let $\eta=\theta-\tau$

$$
v(t)=\left[v(0)+\int_{-\tau}^{t-\tau} e^{\gamma(\eta+\tau)} \beta b \frac{x(\eta) v(\eta)}{1+\alpha v(\eta)} d \eta\right] e^{-\gamma t} .
$$

Since $x(t) \geq 0, v(t) \geq 0$, and $v(0)>0$ for $-\tau \leq t \leq 0$, then $v(t)>0$ for $0 \leq t<\tau$.

Further, when $\tau \leq t<2 \tau$, we have

$$
\begin{aligned}
v(t)=\left\{v(0)+\int_{-\tau}^{0} e^{\gamma(\eta+\tau)} \beta b \frac{x(\eta) v(\eta)}{1+\alpha v(\eta)} d \eta\right. \\
\left.+\int_{0}^{t-\tau} e^{\gamma(\eta+\tau)} \beta b \frac{x(\eta) v(\eta)}{1+\alpha v(\eta)} d \eta\right\} e^{-\gamma t} .
\end{aligned}
$$

By the fact that $x(\eta) \geq 0$ and $v(\eta) \geq 0$ for $-\tau \leq \eta<0$, then

$$
\int_{-\tau}^{0} e^{\gamma(\eta+\tau)} \beta b \frac{x(\eta) v(\eta)}{1+\alpha v(\eta)} d \eta \geq 0
$$

Also $x(\eta)>0$ and $v(\eta)>0$ for $0 \leq \eta<\tau$; then

$$
\int_{0}^{t-\tau} e^{\gamma(\eta+\tau)} \beta b \frac{x(\eta) v(\eta)}{1+\alpha v(\eta)} d \eta>0
$$

Consequently, $v(t)>0$ for $\tau \leq t<2 \tau$, which implies that $v(t)>0$ holds true for $0 \leq t<2 \tau$.

We assume that for a positive integer $k, v(t)>0$ for $0 \leq$ $t<k \tau$. When $k \tau \leq t<(k+1) \tau$, we have

$$
\begin{aligned}
v(t)=\left\{v(0)+\int_{-\tau}^{(k-1) \tau} e^{\gamma(\eta+\tau)} \beta b \frac{x(\eta) v(\eta)}{1+\alpha v(\eta)} d \eta\right. \\
\left.+\int_{(k-1) \tau}^{t-\tau} e^{\gamma(\eta+\tau)} \beta b \frac{x(\eta) v(\eta)}{1+\alpha v(\eta)} d \eta\right\} e^{-\gamma t} .
\end{aligned}
$$

Then similar discussions show that $v(t)>0$ for $k \tau \leq t<$ $(k+1) \tau$. Hence, $v(t)>0$ for all $t>0$.

Theorem 2. All the solutions $(x(t), v(t))^{T}$ of (8) under the initial conditions are ultimately bounded.
Proof. For any solution $(x(t), v(t))^{T}$ of (8), define a function $f(t)=b x(t-\tau)+v(t)$. Then the derivative of $f(t)$ is

$$
\begin{aligned}
\frac{d f(t)}{d t}= & b \frac{d x(t-\tau)}{d t}+\frac{d v(t)}{d t} \\
= & b \lambda-b d x(t-\tau)-\beta b \frac{x(t-\tau) v(t-\tau)}{1+\alpha v(t-\tau)} \\
& +\beta b \frac{x(t-\tau) v(t-\tau)}{1+\alpha v(t-\tau)}-\gamma v(t) \\
= & b \lambda-b d x(t-\tau)-\gamma v(t) \\
\leq & b \lambda-\rho f(t),
\end{aligned}
$$

where $\rho=\min \{d, \gamma\}$.

Integrating both sides of inequality above from 0 to $t$, we have

$$
f(t) \leq \frac{b \lambda}{\rho}+\left(f(0)-\frac{b \lambda}{\rho}\right) e^{-\rho t} .
$$

It means that $b x_{t}(-\tau)+v_{t}(0) \leq b \lambda / \rho$ for any $t \geq 0$ as long as $b \varphi_{1}(-\tau)+\varphi_{2}(0) \leq b \lambda / \rho$. Also,

$$
\limsup _{t \rightarrow \infty} f(t) \leq \frac{b \lambda}{\rho} .
$$

From the first equation of (8), we have

$$
\frac{d x(t)}{d t} \leq \lambda-d x(t) .
$$

Similar discussion shows that

$$
x(t) \leq \frac{\lambda}{d}+\left(x(0)-\frac{\lambda}{d}\right) e^{-d t} .
$$

Then $x_{t}(0) \leq \lambda / d$ for any $t \geq 0$ as long as $\varphi_{1}(0) \leq \lambda / d$. Moreover,

$$
\limsup _{t \rightarrow \infty} x(t) \leq \frac{\lambda}{d}
$$

Thus, the region

$$
\Omega=\left\{\left(\varphi_{1}, \varphi_{2}\right) \in C^{+}: \varphi_{1}(0) \leq \frac{\lambda}{d}, b \varphi_{1}(-\tau)+\varphi_{2}(0) \leq \frac{b \lambda}{\rho}\right\}
$$

is an invariant set and an attractor of system (8) with initial condition $\left(\varphi_{1}, \varphi_{2}\right) \in C^{+}$.

In what follows, we study the existence of equilibria. We consider algebraic equations

$$
\begin{gathered}
\lambda-d x-\frac{\beta x v}{1+\alpha v}=0, \\
\beta b \frac{x v}{1+\alpha v}-\gamma v=0 .
\end{gathered}
$$


It is easy to see that system (24) always has an infectionfree equilibrium $E_{1}(\lambda / d, 0)$. To find the other equilibrium, we assume $v \neq 0$. By the second equation of (24), we have

$$
\frac{\beta b x}{1+\alpha v}=\gamma \text {. }
$$

Then

$$
x=\frac{\gamma(1+\alpha v)}{\beta b} .
$$

We put $x$ into the first equation of (24); then

$$
\begin{gathered}
\lambda-\frac{d \gamma(1+\alpha v)}{\beta b}-\frac{\beta \gamma(1+\alpha v) v}{\beta b(1+\alpha v)}=0, \\
\beta b \lambda-d \gamma=(d \gamma \alpha+\beta \gamma) v .
\end{gathered}
$$

Thus the positive root $E_{2}$ exists if and only if $\beta b \lambda-d \gamma>0$. For system (8), define the basic reproduction number [10] as follows

$$
R_{0}=\frac{\beta b \lambda}{d \gamma} .
$$

It is easy to see that

(i) If $R_{0} \leq 1$, then system (8) has a unique equilibrium $E_{1}(\lambda / d, 0)$, which corresponds to the case that viruses die out, and it is called infection-free equilibrium.

(ii) If $R_{0}>1$, then system ( 8 ) has two equilibria, one is the infection-free equilibrium $E_{1}(\lambda / d, 0)$ and the other is a positive equilibrium $E_{2}\left((\gamma+\alpha b \lambda) / b(d \alpha+\beta), d\left(R_{0}-\right.\right.$ $1) /(d \alpha+\beta))$.

\section{Stability of the Equilibrium}

In this section, we consider the stability of the equilibrium. There are two cases, $\tau=0$ and $\tau>0$.

3.1. Local Stability of Equilibria. First we consider the case of $\tau=0$. In this case system (8) is reduced to a system of ordinary differential equations. In order to examine local stability of an equilibrium, we should compute the eigenvalues of the linearized operator for system (8) at the equilibrium.

By a direct computation, the Jacobian matrix is as follows:

$$
\left(\begin{array}{cc}
-d-\frac{\beta v}{1+\alpha v}, & -\frac{\beta x}{(1+\alpha v)^{2}} \\
\beta b \frac{v}{1+\alpha v}, & \beta b \frac{x}{(1+\alpha v)^{2}}-\gamma
\end{array}\right) .
$$

Consider infection-free equilibrium $E_{1}(\lambda / d, 0)$. The characteristic equation is obtained by the standard method as follows.

It is obvious that $\mu_{1}=-d<0$, and $\mu_{2}=\beta b \lambda / d-\gamma=$ $\gamma\left(R_{0}-1\right)$ are the characteristic roots of the characteristic equation. Therefore, we have the following theorem.
Theorem 3. (i) If $R_{0}<1$, then infection-free equilibrium $E_{1}(\lambda / d, 0)$ is locally asymptotically stable.

(ii) If $R_{0}>1$, then infection-free equilibrium $E_{1}(\lambda / d, 0)$ is unstable.

(iii) If $R_{0}=1$, then infection-free equilibrium $E_{1}(\lambda / d, 0)$ is degenerated.

Now, local stability of the infection equilibrium $E_{2}((\gamma+$ $\left.\alpha b \lambda) / b(d \alpha+\beta), d\left(R_{0}-1\right) /(d \alpha+\beta)\right)$ is considered. As we know, infection equilibrium $E_{2}\left((\gamma+\alpha b \lambda) / b(d \alpha+\beta), d\left(R_{0}-1\right) /(d \alpha+\right.$ $\beta)$ ) exists if and only if $R_{0}>1$.

Theorem 4. If $R_{0}>1$, then the infection equilibrium $E_{2}$ is locally asymptotically stable.

Proof. Set $f(x, v)=\beta x v /(1+\alpha v)$. Then system (8) at the equilibrium $E_{2}\left(x^{*}, v^{*}\right)$ has Jacobian matrix

$$
A=\left(\begin{array}{cc}
-d-\frac{\partial f}{\partial x}\left(x^{*}, v^{*}\right), & -\frac{\partial f}{\partial v}\left(x^{*}, v^{*}\right) \\
b \frac{\partial f}{\partial x}\left(x^{*}, v^{*}\right), & b \frac{\partial f}{\partial v}\left(x^{*}, v^{*}\right)-\gamma
\end{array}\right) .
$$

A direct computation shows that the characteristic equation is

$$
\begin{aligned}
h(\mu)= & \mu^{2}+\left[d+\gamma+\frac{\partial f}{\partial x}\left(x^{*}, v^{*}\right)-b \frac{\partial f}{\partial v}\left(x^{*}, v^{*}\right)\right] \mu \\
& +d \gamma-b d \frac{\partial f}{\partial v}\left(x^{*}, v^{*}\right)+\gamma \frac{\partial f}{\partial x}\left(x^{*}, v^{*}\right)=0 .
\end{aligned}
$$

By Hurwitz criterion, all of the eigenvalues of characteristic equation have negative real parts if and only if

$$
\begin{aligned}
& d+\gamma+\frac{\partial f}{\partial x}\left(x^{*}, v^{*}\right)-b \frac{\partial f}{\partial v}\left(x^{*}, v^{*}\right)>0, \\
& d \gamma-b d \frac{\partial f}{\partial v}\left(x^{*}, v^{*}\right)+\gamma \frac{\partial f}{\partial x}\left(x^{*}, v^{*}\right)>0 .
\end{aligned}
$$

Indeed,

$$
\begin{aligned}
& d+\gamma+\frac{\partial f}{\partial x}\left(x^{*}, v^{*}\right)-b \frac{\partial f}{\partial v}\left(x^{*}, v^{*}\right) \\
& =\frac{(d+\gamma)\left(1+\alpha v^{*}\right)^{2}+\beta v^{*}\left(1+\alpha v^{*}\right)-b \beta x^{*}}{\left(1+\alpha v^{*}\right)^{2}} \\
& =\frac{\left(\alpha^{2} d+\alpha^{2} \gamma+\alpha \beta\right) v^{* 2}+(2 \alpha d+2 \gamma \alpha+\beta) v^{*}-b \beta x^{*}+d+\gamma}{\left(1+\alpha v^{*}\right)^{2}} \\
& =\frac{\left(\alpha^{2} d+\alpha^{2} \gamma+\alpha \beta\right) v^{* 2}+(2 \alpha d+\gamma \alpha+\beta) v^{*}+d}{\left(1+\alpha v^{*}\right)^{2}}>0, \\
& d \gamma-b d \frac{\partial f}{\partial v}\left(x^{*}, v^{*}\right)+\gamma \frac{\partial f}{\partial x}\left(x^{*}, v^{*}\right) \\
& =\frac{d \gamma\left(1+\alpha v^{*}\right)^{2}-b d \beta x^{*}+\gamma \beta v^{*}+\gamma \beta \alpha v^{* 2}}{\left(1+\alpha v^{*}\right)^{2}} \\
& =\frac{\left(\alpha^{2} d \gamma+\alpha \gamma \beta\right) v^{* 2}+(d \alpha \gamma+\gamma \beta) v^{*}}{\left(1+\alpha v^{*}\right)^{2}}>0 .
\end{aligned}
$$

This implies that all the eigenvalues of characteristic equation have negative real parts. Then the infection equilibrium $E_{2}$ 
is locally asymptotically stable. This completes the proof of theorem.

Now we consider the case $\tau>0$. By linearizing system (8) at the infection-free equilibrium $E_{1}(\lambda / d, 0)$, we obtain the characteristic equation as follows:

$$
(\mu+d)\left(\mu+\gamma-e^{-\mu \tau} \beta b \frac{\lambda}{d}\right)=0 .
$$

It is easy to see that $\mu_{1}=-d<0$; hence we only need to discuss the roots of the following equation:

$$
h(\mu, \tau)=\mu+\gamma-e^{-\mu \tau} \beta b \frac{\lambda}{d}=0 .
$$

Theorem 5. When $\tau>0$, then

(i) If $R_{0}<1$, then the infection-free equilibrium $E_{1}(\lambda / d, 0)$ is locally asymptotically stable.

(ii) If $R_{0}>1$, then the infection-free equilibrium $E_{1}(\lambda / d, 0)$ is unstable.

(iii) If $R_{0}=1$, then the infection-free equilibrium $E_{1}(\lambda / d, 0)$ is degenerated.

Proof. (i) By implicit function theorem for complex variables, we know that the roots of (35) are continuous on the parameter $\tau$.

If $R_{0}<1$, then 0 is not a root of (35) for all $\tau>0$. Note that all complex roots of (35) must come in conjugate pairs and the root of (35) is negative for $\tau=0$. Thus, all roots of (35) have negative real parts for small $\tau$; that is, $0<\tau=1$. Suppose that there exists a positive number $\tau=\tau_{0}$ such that (35) has a pair of purely imaginary roots $\lambda= \pm \omega i$; here $\omega$ is a positive number. We have

$$
\omega i+\gamma-e^{-\omega i \tau_{0}} \beta b \frac{\lambda}{d}=0 .
$$

Then

$$
\begin{aligned}
& \beta b \frac{\lambda}{d} \cos \omega \tau_{0}=\gamma, \\
& \beta b \frac{\lambda}{d} \sin \omega \tau_{0}=-\omega .
\end{aligned}
$$

Summing up the square of both equations in (37) we obtain

$$
\begin{aligned}
\omega^{2} & =\beta^{2} b^{2} \frac{\lambda^{2}}{d^{2}}-\gamma^{2}=\frac{\beta^{2} b^{2} \lambda^{2}-\gamma^{2} d^{2}}{d^{2}}=\frac{R_{0}^{2} d^{2} \gamma^{2}-\gamma^{2} d^{2}}{d^{2}} \\
& =\gamma^{2}\left(R_{0}^{2}-1\right) .
\end{aligned}
$$

When $R_{0}<1$, then $\omega^{2}<0$. It is a contradiction with $\omega^{2}>$ 0 which leads to the nonexistence of $\tau_{0}$. This contradiction proves the result.

(ii) When $\mu=0$, and $R_{0}>1$, then

$$
\begin{gathered}
h(0, \tau)=\gamma-\beta b \frac{\lambda}{d}=\gamma\left(1-R_{0}\right)<0, \\
\lim _{\mu \rightarrow \infty} h(\mu, \tau)=+\infty .
\end{gathered}
$$

Therefore equation must have a positive real root for all $\tau>0$. (iii) If $R_{0}=1$, it is easy to know that $\mu=0$ is a root of (35) for all $\tau>0$, which leads to conclusion. (iii) This completes the proof of theorem.

Now we consider the local stability of the infection equilibrium $E_{2}\left((\gamma+\alpha b \lambda) / b(d \alpha+\beta), d\left(R_{0}-1\right) /(d \alpha+\beta)\right)$. As we know, the infection equilibrium $E_{2}\left((\gamma+\alpha b \lambda) / b(d \alpha+\beta), d\left(R_{0}-\right.\right.$ $1) /(d \alpha+\beta))$ exists if and only if $R_{0}>1$. By computation, the associated transcendental characteristic equation of (8) at $E_{2}$ becomes

$$
\mu^{2}+A \mu+B-(C \mu+D) e^{-\mu \tau}=0,
$$

where

$$
\begin{aligned}
A=d+\gamma+\frac{\partial f}{\partial x}\left(x^{*}, v^{*}\right), & B=\left(d+\frac{\partial f}{\partial x}\left(x^{*}, v^{*}\right)\right) \gamma, \\
C=b \frac{\partial f}{\partial v}\left(x^{*}, v^{*}\right), & D=d b \frac{\partial f}{\partial v}\left(x^{*}, v^{*}\right) .
\end{aligned}
$$

Theorem 6. When $\tau>0$, if $R_{0}>1$, then the infection equilibrium $E_{2}$ is locally asymptotically stable.

Proof. By implicit function theorem for complex variables, we know that the root of (40) is continuous on the parameter $\tau$. If $R_{0}>1$, then all roots of (40) have negative real parts as $\tau=0$ and (40) has no zero root for all $\tau>0$. Thus, all roots of (40) have negative real parts for very small $\tau$; that is, $0<\tau \ll 1$. Assume that there exists a positive $\tau_{0}$ such that (40) has a pair of purely imaginary roots $\pm \omega i, \omega>0$. Then $\omega>0$ must satisfy

$$
-\omega^{2}+A \omega i+B-(C \omega i+D)\left(\cos \omega \tau_{0}-i \sin \omega \tau_{0}\right)=0
$$

Separating the real and imaginary parts, we have

$$
\begin{gathered}
C \omega \sin \omega \tau_{0}+D \cos \omega \tau_{0}=B-\omega^{2}, \\
D \sin \omega \tau_{0}-C \omega \cos \omega \tau_{0}=-A \omega
\end{gathered}
$$

which implies that

$$
\omega^{4}+\left(A^{2}-2 B-C^{2}\right) \omega^{2}+B^{2}-D^{2}=0 .
$$

Direct computation shows that

$$
\begin{aligned}
B^{2}-D^{2}= & {\left[\gamma\left(d+\frac{\partial f}{\partial x}\left(x^{*}, v^{*}\right)\right)\right]^{2}-\left[d b \frac{\partial f}{\partial v}\left(x^{*}, v^{*}\right)\right]^{2} } \\
= & {\left[\gamma \frac{\partial f}{\partial x}\left(x^{*}, v^{*}\right)+\gamma d-d b \frac{\partial f}{\partial v}\left(x^{*}, v^{*}\right)\right] } \\
& \times\left[\gamma \frac{\partial f}{\partial x}\left(x^{*}, v^{*}\right)+\gamma d+d b \frac{\partial f}{\partial v}\left(x^{*}, v^{*}\right)\right]>0,
\end{aligned}
$$




$$
\begin{aligned}
A^{2}-2 B-C^{2}= & d^{2}+\gamma^{2}+\left(\frac{\partial f}{\partial x}\left(x^{*}, v^{*}\right)\right)^{2}+2 d \frac{\partial f}{\partial x}\left(x^{*}, v^{*}\right) \\
& -b^{2}\left(\frac{\partial f}{\partial v}\left(x^{*}, v^{*}\right)\right)^{2} \\
= & \left(d+\frac{\partial f}{\partial x}\left(x^{*}, v^{*}\right)\right)^{2} \\
& -b^{2}\left(\frac{\partial f}{\partial v}\left(x^{*}, v^{*}\right)\right)^{2}+\gamma^{2} \\
= & \left(\left(d^{2}+\gamma^{2}\right)\left(1+\alpha v^{*}\right)^{4}+\beta^{2}\left(v^{*}\right)^{2}\left(1+\alpha v^{*}\right)^{2}\right. \\
& \left.+2 d \beta v^{*}\left(1+\alpha v^{*}\right)^{3}-b^{2} \beta^{2}\left(x^{*}\right)^{2}\right) \\
& \times\left(\left(1+\alpha v^{*}\right)^{4}\right)^{-1} .
\end{aligned}
$$

Let

$$
\begin{aligned}
H\left(x^{*}, v^{*}\right)= & \left(d^{2}+\gamma^{2}\right)\left(1+\alpha v^{*}\right)^{4}+\beta^{2}\left(v^{*}\right)^{2}\left(1+\alpha v^{*}\right)^{2} \\
& +2 d \beta v^{*}\left(1+\alpha v^{*}\right)^{3}-b^{2} \beta^{2}\left(x^{*}\right)^{2} .
\end{aligned}
$$

Note that $1+\alpha v^{*}=(\beta b / \gamma) x^{*}$,

$$
\begin{aligned}
& H\left(x^{*}, v^{*}\right) \\
& =\left(d^{2}+\gamma^{2}\right) \frac{\beta^{4} b^{4}}{\gamma^{4}}\left(x^{*}\right)^{4}+\beta^{2}\left(v^{*}\right)^{2} \frac{\beta^{2} b^{2}}{\gamma^{2}}\left(x^{*}\right)^{2} \\
& +2 d \beta v^{*} \frac{\beta^{3} b^{3}}{\gamma^{3}}\left(x^{*}\right)^{3}-\beta^{2} b^{2}\left(x^{*}\right)^{2} \\
& =\left(x^{*}\right)^{2}\left[\frac{\beta^{4} b^{2}}{\gamma^{4}}\left(b^{2} d^{2}\left(x^{*}\right)^{2}+\gamma^{2}\left(v^{*}\right)^{2}+2 \gamma d b v^{*} x^{*}\right)\right. \\
& \left.+\frac{\beta^{4} b^{4}}{\gamma^{2}}\left(x^{*}\right)^{2}-b^{2} \beta^{2}\right] \\
& =\left(x^{*}\right)^{2}\left[\frac{\beta^{4} b^{2}}{\gamma^{4}}\left(b d x^{*}+\gamma v^{*}\right)^{2}+\frac{\beta^{2} b^{2}}{\gamma^{2}}\left(\beta^{2} b^{2}\left(x^{*}\right)^{2}-\gamma^{2}\right)\right] \\
& =\left(x^{*}\right)^{2}\left[\frac{\beta^{4} b^{2}}{\gamma^{4}}\left(b d x^{*}+\gamma v^{*}\right)^{2}+\beta^{2} b^{2}\left(\alpha^{2}\left(v^{*}\right)^{2}+2 \alpha v^{*}\right)\right]
\end{aligned}
$$$$
>0 \text {. }
$$

By Hurwitz criterion, (44) has no positive roots, which implies the nonexistence of $\tau_{0}$. Thus all roots of (40) have negative real parts for $\tau>0$.

3.2. Global Stability of Equilibria. In the section, we study the global stability of equilibria; we first consider the infectionfree equilibrium $E_{1}$.

Theorem 7. When $\tau=0$,

(i) If $R_{0} \leq 1$, then infection-free equilibrium $E_{1}(\lambda / d, 0)$ is globally asymptotically stable. (ii) If $R_{0}>1$, then infection equilibrium $E_{2}\left(x^{*}, v^{*}\right)$ is globally asymptotically stable.

Proof. (i) Define a Lyapunov function as what follows

$$
\begin{gathered}
\quad V(x, v)=x-\frac{\lambda}{d} \ln x+\frac{v}{b}+\frac{\lambda}{d} \ln \frac{\lambda}{d}-\frac{\lambda}{d} \\
\left.\frac{d V(x, v)}{d t}\right|_{(8)} \\
=\frac{x-\lambda / d}{x}\left(\lambda-d x-\frac{\beta x v}{1+\alpha v}\right)+\frac{1}{b}\left(\frac{\beta b x v}{1+\alpha v}-\gamma v\right) \\
=-\frac{(d x-\lambda)^{2}}{d x}+\frac{\beta x v}{1+\alpha v}\left(1-\frac{d x-\lambda}{d x}\right)-\frac{\gamma}{b} v \\
=-\frac{(d x-\lambda)^{2}}{d x}+\left(\frac{\beta \lambda}{d(1+\alpha v)}-\frac{\gamma}{b}\right) v \\
=-\frac{(d x-\lambda)^{2}}{d x}+\frac{\gamma}{b}\left(R_{0}-1\right) v-\frac{\beta \gamma}{d} \frac{\alpha v^{2}}{1+\alpha v} .
\end{gathered}
$$

It means that $d V(x, v) /\left.d t\right|_{(8)}$ is negative semidefinite as $R_{0} \leq$ 1. Moreover, the last equality of the above equation shows that the largest invariant set of system (8) on the region $\left\{(x, v)^{T} \in \mathbb{R}_{+}^{2}: d V / d t=0\right\}$ is the singleton $\left\{E_{1}\right\}$. Therefore, the infection-free equilibrium $E_{1}$ is global asymptotically stability.

(ii) We rewrite the system (8)

$$
\begin{aligned}
& \frac{d x(t)}{d t}=\lambda-d x(t)-\frac{\beta x(t) v(t)}{1+\alpha v(t)}=Q(x, v), \\
& \frac{d v(t)}{d t}=\beta b \frac{x(t) v(t)}{1+\alpha v(t)}-\gamma v(t)=P(x, v) .
\end{aligned}
$$

Choose a Dulac function

$$
D(x, v)=\frac{1+\alpha v}{\beta v}
$$

We have

$$
\frac{\partial(D P)}{\partial v}+\frac{\partial(D Q)}{\partial x}=-\frac{\gamma \alpha}{\beta}-d \frac{1+\alpha v}{\beta v}-1<0
$$

Thus system (49) does not have nontrivial periodic orbits in $\Omega$. The conclusion follows.

Theorem 8. When $\tau>0$, if $R_{0} \leq 1$, then infection-free equilibrium $E_{1}(\lambda / d, 0)$ is globally asymptotically stable. 
Proof. Define a functional $g: C^{+} \rightarrow \mathbb{R}$ as follows:

$$
\begin{aligned}
g\left(\varphi_{1}, \varphi_{2}\right)= & \frac{1}{2}\left[\varphi_{1}(0)-\frac{\lambda}{d}\right]^{2} \\
& +\frac{\lambda}{d}\left[\varphi_{2}(0)+\beta b \int_{-\tau}^{0} \frac{\varphi_{1}(\theta) \varphi_{2}(\theta)}{1+\alpha \varphi_{2}(\theta)} d \theta\right] .
\end{aligned}
$$

For any $t \geq 0, x_{t}, v_{t} \in C$, then

$$
\begin{aligned}
& g\left(x_{t}, v_{t}\right)=\frac{b}{2}\left[x(t)-\frac{\lambda}{d}\right]^{2} \\
& +\frac{\lambda}{d}\left[v(t)+\beta b \int_{t-\tau}^{t} \frac{x(\theta) v(\theta)}{1+\alpha v(\theta)} d \theta\right], \\
& \frac{d g\left(x_{t}, v_{t}\right)}{d t}=b\left(x(t)-\frac{\lambda}{d}\right) \frac{d x(t)}{d t}+\frac{\lambda}{d} \frac{d v(t)}{d t} \\
& +\frac{\beta b \lambda}{d}\left[\frac{x(t) v(t)}{1+\alpha v(t)}-\frac{x(t-\tau) v(t-\tau)}{1+\alpha v(t-\tau)}\right] \\
& =b\left[x(t)-\frac{\lambda}{d}\right]\left[\lambda-d x(t)-\frac{\beta x(t) v(t)}{1+\alpha v(t)}\right] \\
& +\frac{\lambda}{d}\left[\beta b \frac{x(t) v(t)}{1+\alpha v(t)}-\gamma v(t)\right] \\
& =-b d\left[x(t)-\frac{\lambda}{d}\right]^{2}-\beta b \frac{x(t) v(t)}{1+\alpha v(t)}\left[x(t)-\frac{\lambda}{d}\right] \\
& +\frac{\lambda}{d}\left[\beta b \frac{x(t) v(t)}{1+\alpha v(t)}-\gamma v(t)\right] \\
& =-b\left(d+\frac{\beta v(t)}{1+\alpha v(t)}\right)\left[x(t)-\frac{\lambda}{d}\right]^{2} \\
& +\frac{\lambda v(t)}{d}\left(\frac{\beta b \lambda}{d(1+\alpha v(t))}-\gamma\right) \\
& =-b\left(d+\frac{\beta v(t)}{1+\alpha v(t)}\right)\left[x(t)-\frac{\lambda}{d}\right]^{2} \\
& +\frac{\lambda v(t) \gamma}{d}\left(\frac{R_{0}}{1+\alpha v(t)}-1\right) \\
& =-b\left(d+\frac{\beta v(t)}{1+\alpha v(t)}\right)\left[x(t)-\frac{\lambda}{d}\right]^{2} \\
& +\frac{\lambda v(t) \gamma}{d}\left(R_{0}-1\right)-\frac{\alpha v(t) R_{0}}{1+\alpha v(t)} \\
& \leq-b\left(d+\frac{\beta v(t)}{1+\alpha v(t)}\right)\left[x(t)-\frac{\lambda}{d}\right]^{2} \\
& +\frac{\lambda v(t) \gamma}{d}\left(R_{0}-1\right) \text {. }
\end{aligned}
$$

Then,

$$
\begin{aligned}
\left.\frac{d g\left(\varphi_{1}, \varphi_{2}\right)}{d t}\right|_{(8)}= & -b\left(d+\frac{\beta \varphi_{2}(0)}{1+\alpha \varphi_{2}(0)}\right)\left[\varphi_{1}(0)-\frac{\lambda}{d}\right]^{2} \\
& +\frac{\lambda \varphi_{2}(0) \gamma}{d}\left(R_{0}-1\right)-\frac{\alpha \varphi_{2}(0) R_{0}}{1+\alpha \varphi_{2}(0)}
\end{aligned}
$$

When $R_{0} \leq 1$, we have $d g\left(x_{t}, v_{t}\right) / d t \leq 0$. It is easy to know, when $R_{0} \leq 1$ the largest invariant set of system (8) on the region $\left\{\left(\varphi_{1}, \varphi_{2}\right) \in C^{+}: d g\left(\varphi_{1}, \varphi_{2}\right) / d t=0\right\}$ is the singleton $\left\{E_{1}\right\}$. By Lassalle invariant principle for autonomous retarded differential equations [11], infection-free equilibrium $E_{1}$ is globally asymptotically stable. This completes the proof.

Theorem 9. When $\tau>0$, if $R_{0}>1$, then the infection equilibrium $E_{2}\left(x^{*}, v^{*}\right)$ is globally asymptotically stable.

Proof. Let $V(t)=v(t+\tau)$ and $X(t)=x(t)$; system (8) becomes

$$
\begin{aligned}
& \frac{d X(t)}{d t}=\lambda-d X(t)-\frac{\beta X(t) V(t-\tau)}{1+\alpha V(t-\tau)}, \\
& \frac{d V(t)}{d t}=\beta b \frac{X(t) V(t-\tau)}{1+\alpha V(t-\tau)}-\gamma V(t) .
\end{aligned}
$$

Denote $u(x)=x /(1+\alpha x)$. Evaluating both sides of (55) at $E_{2}$, we obtain

$$
\lambda=d x^{*}+\beta x^{*} u\left(v^{*}\right), \quad \gamma v^{*}=\beta b x^{*} u\left(v^{*}\right) .
$$

Define a Lyapunov functional $L: C^{+} \rightarrow \mathbb{R}$ as follows:

$$
\begin{aligned}
L\left(\varphi_{1}, \varphi_{2}\right)= & \frac{1}{\beta u\left(v^{*}\right)} L_{1}\left(\varphi_{1}, \varphi_{2}\right)+\frac{v^{*}}{\beta b x^{*} u\left(v^{*}\right)} L_{2}\left(\varphi_{1}, \varphi_{2}\right) \\
& +L_{3}\left(\varphi_{1}, \varphi_{2}\right),
\end{aligned}
$$

where

$$
\begin{aligned}
& L_{1}\left(\varphi_{1}, \varphi_{2}\right)=\frac{\varphi_{1}(0)}{x^{*}}-1-\ln \frac{\varphi_{1}(0)}{x^{*}} \\
& L_{2}\left(\varphi_{1}, \varphi_{2}\right)=\frac{\varphi_{2}(0)}{v^{*}}-1-\ln \frac{\varphi_{2}(0)}{v^{*}} \\
& L_{3}\left(\varphi_{1}, \varphi_{2}\right)=\int_{-\tau}^{0}\left(\frac{\varphi_{2}(\theta)}{v^{*}}-1-\ln \frac{\varphi_{2}(\theta)}{v^{*}}\right) d \theta .
\end{aligned}
$$

Thus, $L\left(\varphi_{1}, \varphi_{2}\right) \geq 0$ with equality if and only if $\varphi_{1}(0) / x^{*}=$ $\varphi_{2}(0) / v^{*}=1$.

For any $t \geq 0, X_{t}, V_{t} \in C$, then

$$
\begin{gathered}
L_{1}\left(X_{t}, V_{t}\right)=\frac{X(t)}{x^{*}}-1-\ln \frac{X(t)}{x^{*}}, \\
L_{2}\left(X_{t}, V_{t}\right)=\frac{V(t)}{v^{*}}-1-\ln \frac{V(t)}{v^{*}}, \\
L_{3}\left(X_{t}, V_{t}\right)=\int_{-\tau}^{0}\left(\frac{V(t+\theta)}{v^{*}}-1-\ln \frac{V(t+\theta)}{v^{*}}\right) d \theta .
\end{gathered}
$$


We calculate derivatives of $L_{1}\left(X_{t}, V_{t}\right), L_{2}\left(X_{t}, V_{t}\right)$, and $L_{3}\left(X_{t}, V_{t}\right)$ with respect to the system (55):

$$
\begin{aligned}
& \frac{d L_{1}\left(X_{t}, V_{t}\right)}{d t} \\
& =\frac{1}{x^{*}}\left(1-\frac{x^{*}}{X}\right)(\lambda-d X-\beta X u(V(t-\tau))) \\
& =\frac{1}{x^{*}}\left(1-\frac{x^{*}}{X}\right)\left(d x^{*}+\beta x^{*} u\left(v^{*}\right)-d X-\beta X u(V(t-\tau))\right) \\
& =-d \frac{\left(X-x^{*}\right)^{2}}{X x^{*}}+\beta u\left(v^{*}\right)\left(1-\frac{x^{*}}{X}\right)\left(1-\frac{X u(V(t-\tau))}{x^{*} u\left(v^{*}\right)}\right) \\
& =-d \frac{\left(X-x^{*}\right)^{2}}{X x^{*}} \\
& +\beta u\left(v^{*}\right)\left(1-\frac{x^{*}}{X}+\frac{u(V(t-\tau))}{u\left(v^{*}\right)}-\frac{X u(V(t-\tau))}{x^{*} u\left(v^{*}\right)}\right), \\
& \frac{d L_{2}\left(X_{t}, V_{t}\right)}{d t} \\
& =\frac{1}{v^{*}}\left(1-\frac{v^{*}}{V}\right)(\beta b X u(V(t-\tau))-\gamma V) \\
& =\frac{1}{v^{*}}\left(1-\frac{v^{*}}{V}\right)\left(\beta b x^{*} u\left(v^{*}\right) \frac{X u(V(t-\tau))}{x^{*} u\left(v^{*}\right)}-\gamma \frac{V v^{*}}{v^{*}}\right) \\
& =\beta b \frac{x^{*}}{v^{*}} u\left(v^{*}\right)\left(1-\frac{v^{*}}{V}\right)\left(\frac{X}{x^{*}} \frac{u(V(t-\tau))}{u\left(v^{*}\right)}-\frac{V}{v^{*}}\right) \\
& =\beta b \frac{x^{*}}{v^{*}} u\left(v^{*}\right) \\
& \times\left(1-\frac{v^{*}}{V} \frac{X}{x^{*}} \frac{u(V(t-\tau))}{u\left(v^{*}\right)}+\frac{X}{x^{*}} \frac{u(V(t-\tau))}{u\left(v^{*}\right)}-\frac{V}{v^{*}}\right), \\
& \frac{d L_{3}\left(X_{t}, V_{t}\right)}{d t} \\
& =\int_{-\tau}^{0} \frac{d}{d t}\left(\frac{V(t+\theta)}{v^{*}}-1-\ln \frac{V(t+\theta)}{v^{*}}\right) d \theta \\
& =\frac{V(t)}{v^{*}}-\ln \frac{V(t)}{v^{*}}-\frac{V(t-\tau)}{v^{*}}+\ln \frac{V(t-\tau)}{v^{*}} .
\end{aligned}
$$

We obtain

$$
\begin{aligned}
& \frac{d L\left(X_{t}, V_{t}\right)}{d t} \\
& =-\frac{d}{\beta u\left(v^{*}\right)} \frac{\left(X-x^{*}\right)^{2}}{X x^{*}}+1-\frac{x^{*}}{X} \\
& +\frac{u(V(t-\tau))}{u\left(v^{*}\right)}-\frac{X u(V(t-\tau))}{x^{*} u\left(v^{*}\right)}+1-\frac{v^{*}}{V} \frac{X}{x^{*}} \frac{u(V(t-\tau))}{u\left(v^{*}\right)} \\
& +\frac{X}{x^{*}} \frac{u(V(t-\tau))}{u\left(v^{*}\right)}-\frac{V}{v^{*}}+\frac{V}{v^{*}}-\ln \frac{V}{v^{*}}-\frac{V(t-\tau)}{v^{*}}
\end{aligned}
$$

$$
\begin{aligned}
& +\ln \frac{V(t-\tau)}{v^{*}} \\
= & -\frac{d}{\beta u\left(v^{*}\right)} \frac{\left(X-x^{*}\right)^{2}}{X x^{*}}+2-\frac{x^{*}}{X}+\frac{u(V(t-\tau))}{u\left(v^{*}\right)} \\
& -\frac{v^{*}}{V} \frac{X}{x^{*}} \frac{u(V(t-\tau))}{u\left(v^{*}\right)} \\
& -\ln \frac{V}{v^{*}}-\frac{V(t-\tau)}{v^{*}}+\ln \frac{V(t-\tau)}{v^{*}} .
\end{aligned}
$$

By adding and subtracting the quantity $\ln \left(\left(X / x^{*}\right)(u(V(t-\right.$ $\left.\tau)) / u\left(v^{*}\right)\right)$ ), we have

$$
\begin{aligned}
& \frac{d L\left(X_{t}, V_{t}\right)}{d t} \\
& =-\frac{d}{\beta u\left(v^{*}\right)} \frac{\left(X-x^{*}\right)^{2}}{X x^{*}}+2-\frac{x^{*}}{X} \\
& +\frac{u(V(t-\tau))}{u\left(v^{*}\right)}-\frac{v^{*}}{V} \frac{X}{x^{*}} \frac{u(V(t-\tau))}{u\left(v^{*}\right)} \\
& -\frac{V(t-\tau)}{v^{*}}+\ln \frac{V(t-\tau)}{v^{*}}+\ln \left(\frac{X}{x^{*}} \frac{v^{*}}{V} \frac{u(V(t-\tau))}{u\left(v^{*}\right)}\right) \\
& -\ln \left(\frac{X}{x^{*}}\right)-\ln \left(\frac{u(V(t-\tau))}{u\left(v^{*}\right)}\right) \\
& =-\frac{d}{\beta u\left(v^{*}\right)} \frac{\left(X-x^{*}\right)^{2}}{X x^{*}}-\left(\frac{x^{*}}{X}-1+\ln \frac{X}{x^{*}}\right) \\
& -\left(\frac{X}{x^{*}} \frac{v^{*}}{V} \frac{u(V(t-\tau))}{u\left(v^{*}\right)}-1\right. \\
& \left.-\ln \left(\frac{X}{x^{*}} \frac{v^{*}}{V} \frac{u(V(t-\tau))}{u\left(v^{*}\right)}\right)\right) \\
& +\frac{u(V(t-\tau))}{u\left(v^{*}\right)}-\frac{V(t-\tau)}{v^{*}} \\
& +\ln \frac{V(t-\tau)}{v^{*}}-\ln \left(\frac{u(V(t-\tau))}{u\left(v^{*}\right)}\right) .
\end{aligned}
$$

Then,

$$
\begin{aligned}
\frac{d L\left(\varphi_{1}, \varphi_{2}\right)}{d t}= & -\frac{d}{\beta u\left(v^{*}\right)} \frac{\left(\varphi_{1}(0)-x^{*}\right)^{2}}{\varphi_{1}(0) x^{*}} \\
& -\left(\frac{x^{*}}{\varphi_{1}(0)}-1+\ln \frac{\varphi_{1}(0)}{x^{*}}\right) \\
& -\left(\frac{\varphi_{1}(0)}{x^{*}} \frac{v^{*}}{\varphi_{2}(0)} \frac{u\left(\varphi_{2}(-\tau)\right)}{u\left(v^{*}\right)}-1\right. \\
& \left.-\ln \left(\frac{\varphi_{1}(0)}{x^{*}} \frac{v^{*}}{\varphi_{2}(0)} \frac{u\left(\varphi_{2}(-\tau)\right)}{u\left(v^{*}\right)}\right)\right)
\end{aligned}
$$




$$
\begin{aligned}
& +\frac{u\left(\varphi_{2}(-\tau)\right)}{u\left(v^{*}\right)}-\frac{\varphi_{2}(-\tau)}{v^{*}} \\
& +\ln \frac{\varphi_{2}(-\tau)}{v^{*}}-\ln \left(\frac{u\left(\varphi_{2}(-\tau)\right)}{u\left(v^{*}\right)}\right) .
\end{aligned}
$$

It is easy to know that $u\left(\varphi_{2}(-\tau)\right) / u\left(v^{*}\right)-\varphi_{2}(-\tau) / v^{*}+$ $\ln \left(\varphi_{2}(-\tau) / v^{*}\right)-\ln \left(u\left(\varphi_{2}(-\tau)\right) / u\left(v^{*}\right)\right) \leq 0$, and $u\left(\varphi_{2}(-\tau)\right) /$ $u\left(v^{*}\right)-\varphi_{2}(-\tau) / v^{*}+\ln \left(\varphi_{2}(-\tau) / v^{*}\right)-\ln \left(u\left(\varphi_{2}(-\tau)\right) / u\left(v^{*}\right)\right)=0$ if and only if $\varphi_{2}(-\tau) / v^{*}=1$. It follows that $d L\left(\varphi_{1}, \varphi_{2}\right) / d t \leq 0$, and $d L\left(\varphi_{1}, \varphi_{2}\right) / d t=0$ if and only if $\varphi_{1}(0) / x^{*}=\varphi_{2}(0) / v^{*}=$ $\varphi_{2}(-\tau) / v^{*}=1$. By classical stability theory for functional differential equations, $E_{2}$ is globally asymptotically stable. This completes the proof.

\section{Conclusion}

The viral infection model addressed in this paper has saturated incidence rate and viral infection with delay. The basic reproductive number $R_{0}$ is given. When $R_{0}<1$, for the model with or without delay time, the infection-free equilibrium is globally asymptotically stable, which implies that the viral infection goes extinct eventually; when $R_{0}>1$, the infection equilibrium is globally asymptotically stable, which implies that the viral infection persists in the body of the host.

\section{Conflict of Interests}

The author declares that there is no conflict of interests regarding the publication of this paper.

\section{Acknowledgments}

This work was partially supported by National Natural Science Foundation of China (no. 11371107 and no. 11031002), Research Fund for the Doctoral Program of Higher Education of China (no. 20124410110001), and Program for Changjiang Scholars and Innovative Research Team in University (IRT1226).

\section{References}

[1] M. A. Nowak, S. Bonhoeffer, A. M. Hill, R. Boehme, H. C. Thomas, and H. Mcdade, "Viral dynamics in hepatitis B virus infection," Proceedings of the National Academy of Sciences of the United States of America, vol. 93, no. 9, pp. 4398-4402, 1996.

[2] A. Korobeinikov, "Global properties of basic virus dynamics models," Bulletin of Mathematical Biology, vol. 66, no. 4, pp. 879-883, 2004.

[3] A. M. Elaiw, "Global properties of a class of HIV models," Nonlinear Analysis: Real World Applications, vol. 11, no. 4, pp. 2253-2263, 2010.

[4] J. Li, X. Song, and F. Gao, "Global stability of a viral infection model with two delays and two types of target cells," The Journal of Applied Analysis and Computation, vol. 2, no. 3, pp. 281-292, 2012.
[5] R. Qesmi, J. Wu, J. Wu, and J. M. Heffernan, "Influence of backward bifurcation in a model of hepatitis B and C viruses," Mathematical Biosciences, vol. 224, no. 2, pp. 118-125, 2010.

[6] D. Sansonno, A. R. Iacobelli, V. Cornacchiulo et al., "Detection of hepatitis $\mathrm{C}$ virus (HIV) proteins by immunouorescence and HCV RNA genomic sequences by nonisotopic in situ hybridization in bone marrow and peripheral blood mononnuclear cells of chronically HCV infected," Clinical \& Experimental Immunology, vol. 103, pp. 414-421, 1996.

[7] D. Wodarz and D. N. Levy, "Human immunodeficiency virus evolution towards reduced replicative fitness in vivo and the development of AIDS," Proceedings of the Royal Society B: Biological Sciences, vol. 274, no. 1624, pp. 2481-2490, 2007.

[8] X. Zhou, X. Song, and X. Shi, "A differential equation model of HIV infection of $C D 4^{+}$T-cells with cure rate," Journal of Mathematical Analysis and Applications, vol. 342, no. 2, pp. 1342-1355, 2008.

[9] V. Capasso and G. Serio, "A generalization of the KermackMcKendrick deterministic epidemic model," Mathematical Biosciences, vol. 42, no. 1-2, pp. 43-61, 1978.

[10] P. van den Driessche and J. Watmough, "Reproduction numbers and sub-threshold endemic equilibria for compartmental models of disease transmission," Mathematical Biosciences, vol. 180, pp. 29-48, 2002.

[11] J. Hale, Theory of Functional Differential Equations, Springer, New York, NY, USA, 2nd edition, 1977. 


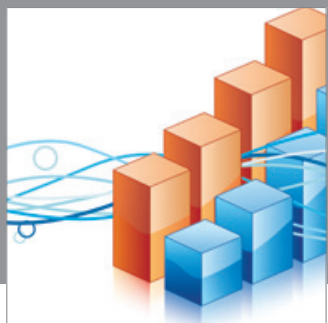

Advances in

Operations Research

mansans

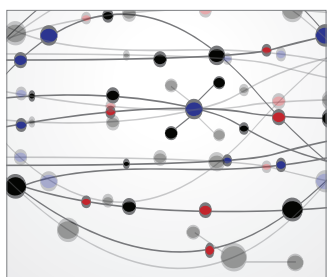

The Scientific World Journal
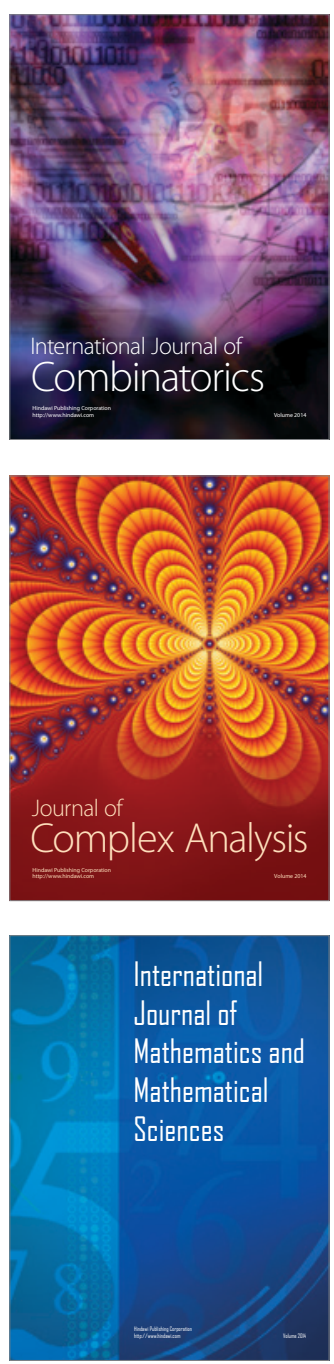
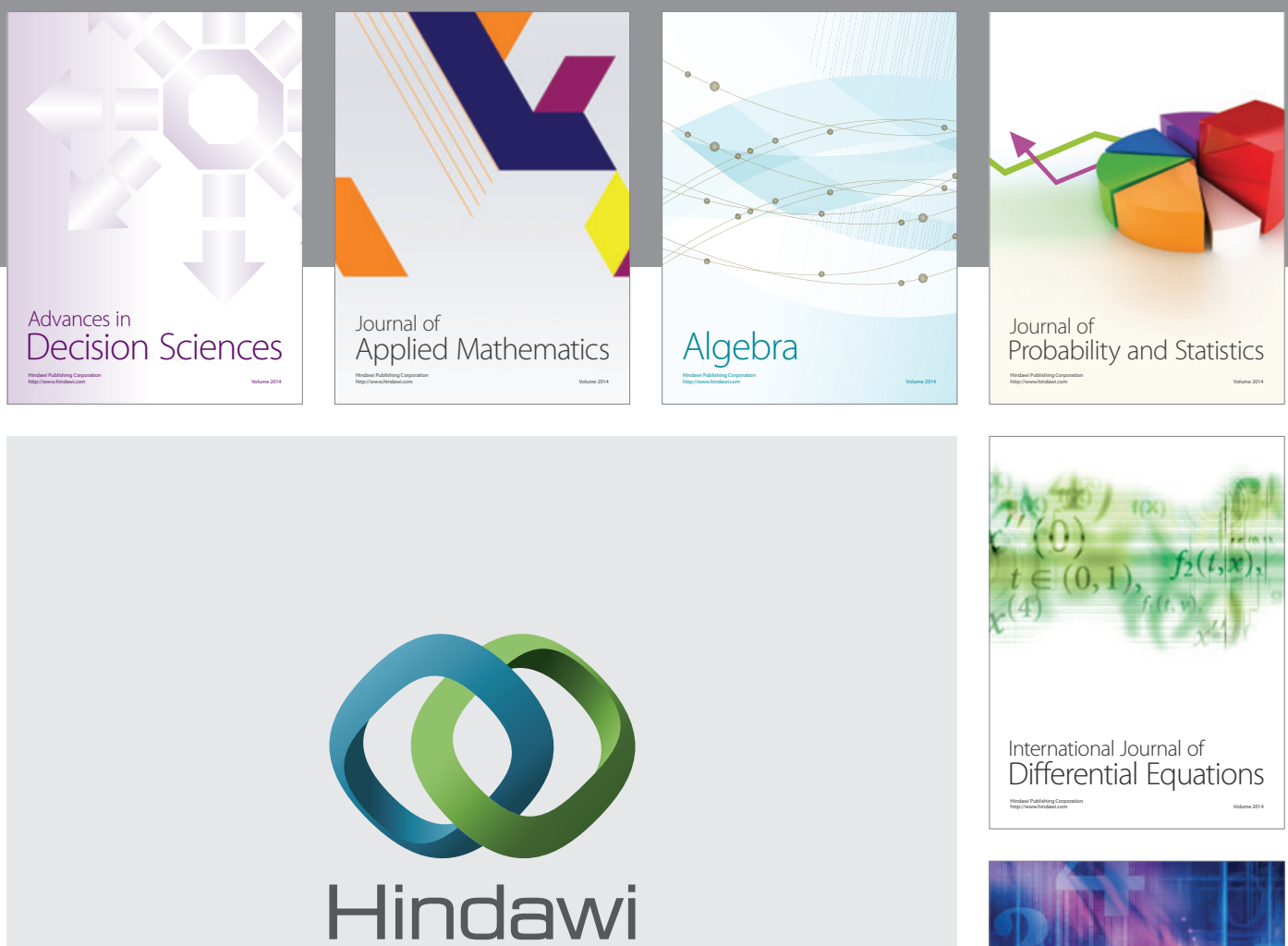

Submit your manuscripts at http://www.hindawi.com
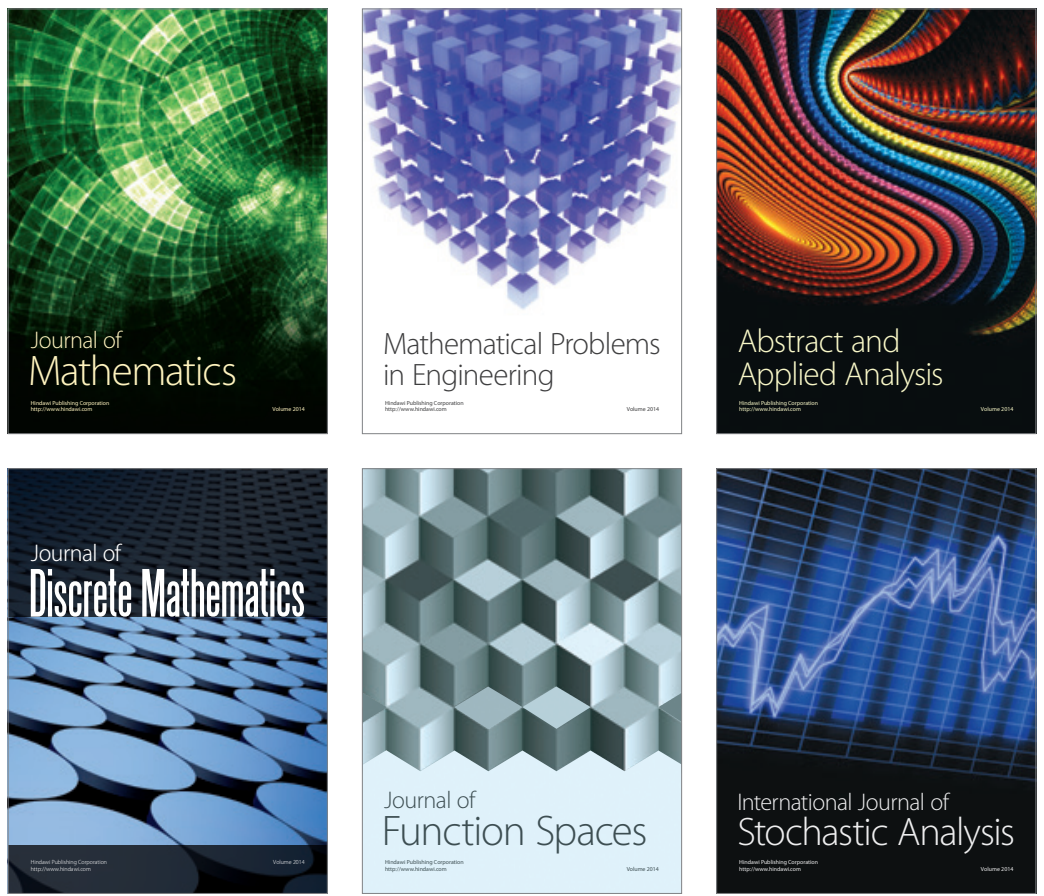

Journal of

Function Spaces

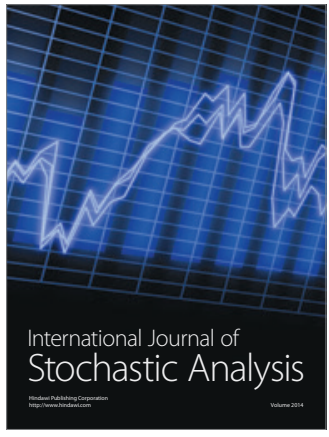

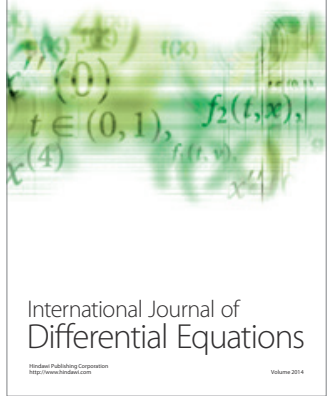
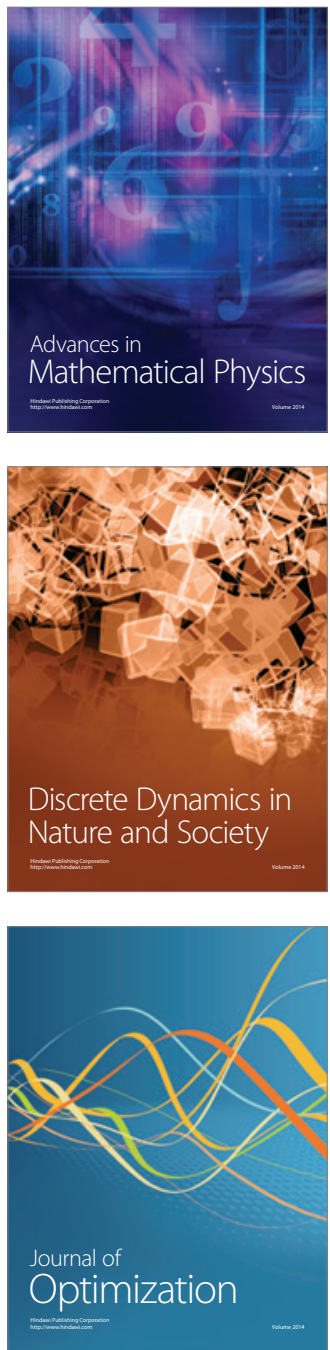